\title{
Fertility preservation in young women with breast cancer
}

\author{
Jennifer R. Klemp • S. Samuel Kim • \\ on behalf of ISFP Practice Committee
}

Received: 19 April 2012 /Accepted: 25 April 2012 / Published online: 22 May 2012

(C) Springer Science+Business Media, LLC 2012

\begin{abstract}
When a young woman is diagnosed with breast cancer, there is often a sense of urgency by the patient and her providers to initiate treatment. This article provides guidelines for incorporating the discussion of fertility preservation with newly diagnosed young women with breast cancer.
\end{abstract}

Keywords Fertility preservation - Breast cancer . Chemotherapy · Cancer · Survivorship · Oocyte cryopreservation · Embryo cryopreservation - Ovarian tissue cryopreservation $\cdot$ Ovarian stimulation $\cdot$ Infertility

Capsule Guidelines for Fertility Preservation in young women with breast cancer.

ISFP Practice Committee: S. Samuel Kim, Jacques Donnez, Pedro Barri, Antonio Pellicer, Pasquale Patrizio, Zev Rosenwaks, Peter Nagy, Tommaso Falcone, Claus Andersen, Outi Hovatta, Hamish Wallace, Dror Meirow, Debra Gook, Seok H Kim, Chii-Ruey Tzeng, Shuetu Suzuki, Bunpei Ishizuka

Statements and opinions expressed in articles and communications herein are those of the author(s) and not necessarily those of the editor, publisher, or the American Society for Reproductive Medicine, or any organizations endorsing this journal. Neither the editor, publisher, nor organizations endorsing this journal, guarantee, warrant, or endorse any product or service advertised in this journal, nor do they guarantee any claim made by the manufacturer of such product or service. Except as noted, authors have reported no competing interests.

\section{J. R. Klemp}

Division of Medical Oncology,

University of Kansas School of Medicine,

Kansas City, KS, USA

\section{S. S. Kim $(\bowtie)$}

Department of OB/GYN, University of Kansas Medical Center,

3901 Rainbow Blvd.,

Kansas City, KS 66212, USA

e-mail: skim2@kumc.edu

\section{Background}

Breast cancer is the most common cancer in women with over 230,000 new diagnosis of invasive breast cancer per year in the United States [1]. With more than 5-7 \% of new breast cancers diagnosed under age $40[2,3]$, we have a growing need to develop best practices for addressing fertility preservation in this population of young women. As a result of early detection and targeted therapies, many of these women will survive, resulting in the need to educate patients and providers about the late and long-term effects of cancer and its treatment including issues related to fertility.

It has previously been reported that female cancer survivors routinely do not receive counseling about fertility preservation prior to initiating their cancer treatment and this has resulted in many experiencing long-term regret and worse overall quality of life [4].

The challenge is incorporating this discussion into an already intense, complicated and emotional time for young woman newly diagnosed with breast cancer. In 2006, the American Society of Clinical Oncology (ASCO) [5] published recommendations on fertility preservation and included this statement, "As part of education and informed consent prior to cancer therapy, oncologists should address the possibility of infertility with patients treated during their reproductive years and be prepared to discuss possible fertility preservation options or refer appropriate and interested patients to reproductive specialists". However, it was subsequently reported that fertility preservation is not a priority for oncologists [6]. This may be due to a lack of awareness on options for fertility preservation, access to resources and fertility specialists, and insurance reimbursement concerns.

With professional recommendations from ASCO, the American Society of Reproductive Medicine, the International Society for Fertility Preservation, web-based resources, and 
Table 1 Methods for fertility preservation in young women with breast cancer

\begin{tabular}{ll}
\hline Method & Description \\
\hline Embryo Cryopreservation & $\begin{array}{c}\text { ovarian stimulation, harvesting eggs, in vitro fertilization, and } \\
\text { freezing of embryos } \\
\text { ovarian stimulation, harvesting eggs, and freezing of mature eggs } \\
\text { harvesting ovarian tissue surgically, freezing of ovarian tissue } \\
\text { Ovarian Tissue Cryopreservation } \\
\text { Ovarian Suppression with GnRHa }\end{array}$ \\
\hline
\end{tabular}

publications highlighting research and practice guidelines, there are now tools available for improving access to education and resources.

\section{What are the goals of fertility preservation?}

It is important to understand even if cyclic menses resumes, fertility may be compromised post treatment. Therefore, it is essential for patients to receive education on topics related to methods for preserving fertility, based on many factors including age, ovarian reserve, treatment, time, and partner availability. Part of the informed decision making process for newly diagnosed patients include the late and long-term side effects of their recommended treatment and impact on fertility and options for preservation (Table 1) should be a standard part of that discussion.

Recent publications have highlighted the incorporation of fertility preservation into practice for young women newly diagnosed with breast cancer. These algorithms demonstrate the need for informed decision making from the time of diagnosis depending on breast cancer subtype and treatment regimen [7], adequate ovarian reserve [8], adequate time prior to initiating treatment and access to self or donor egg and partner or donor sperm [9], along with the patient's age, prognosis, the toxicity of the treatment regimen recommen- ded, and taking into account the patient's individual choice to undergo fertility preservation [10]. These algorithms provide a useful reference for busy oncologists and their treatment team. In addition, consultation postadjuvant treatment may also be an opportunity for young women with breast cancer to discuss their potential ovarian reserve and should be considered in women who did not undergo pre-treatment consultation. Figures 1 and 2 highlight the incorporation of discussions around fertility preservation at the time of diagnosis or in post-treatment care.

\section{When to refer the patient?}

In an ideal situation, a fertility specialist should counsel women at the time of a cancer diagnosis. If a woman is considering future fertility, an immediate referral to a fertility specialist should be made prior to the initiation of cancer treatment. Ovarian stimulation for 2-3 weeks is usually required for oocyte cryopreservation and embryo cryopreservation. For women who require immediate cancer treatment (such as those who require neoadjuvant chemotherapy) or those who are not good candidates for ovarian stimulation, ovarian tissue cryopreservation may be an option. The best time for fertility preservation is after surgery and before adjuvant chemotherapy.
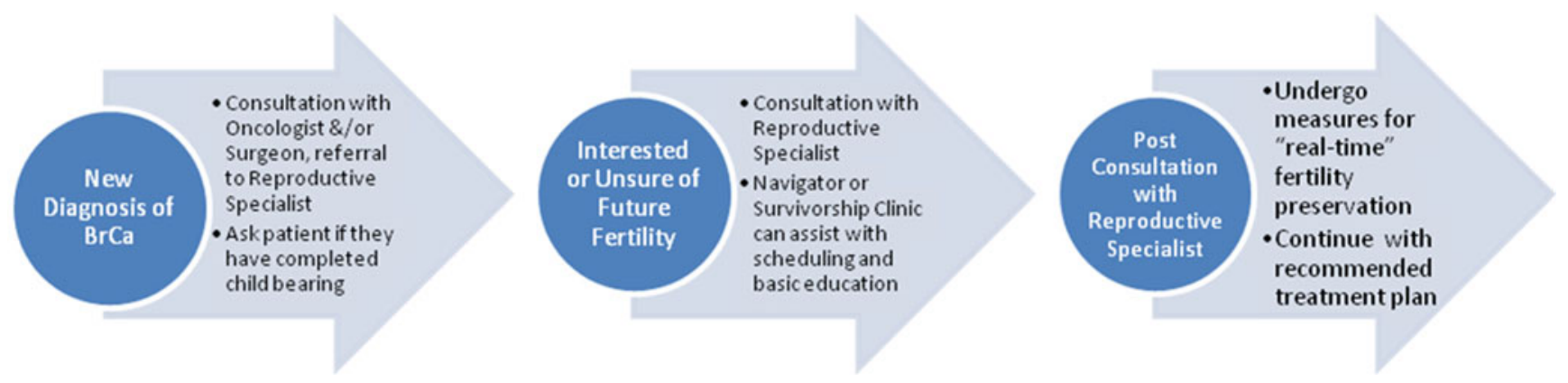

Fig. 1 Young women with breast cancer at the time of diagnosis 

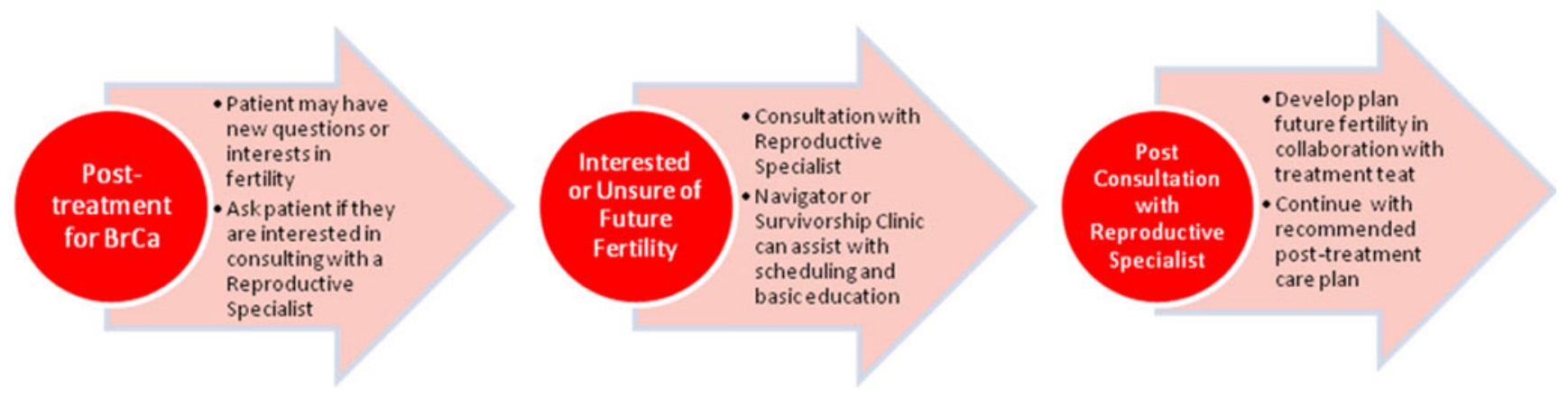

Fig. 2 Young women with breast cancer post-treatment

\section{What to consider for fertility preservation?}

The rate of ovarian failure following breast cancer treatment is dependent on multiple factors including the age of the patient, ovarian reserve, dosage and type of chemotherapy, and number of cycles of chemotherapy, and ranges from $18-61 \%$. For example, if the age of the patients is over $37 \mathrm{y} / \mathrm{o}$ with a low ovarian reserve, the chance of preserving fertility is low. In that case, the risk/benefit ratio may not favor fertility preservation. As a rule of thumb, ovarian tissue cryopreservation is recommended if the patient requires immediate chemotherapy or has rapidly growing estrogen dependent tumor. Otherwise, embryo cryopreservation (for those who have a partner) or oocyte cryopreservation should be considered as the first line of strategy. The risk of controlled ovarian stimulation in breast cancer patients (especially ER+) is unknown. Although short-term exposure to high concentrations of estrogen may not have adverse effects on tumor growth, it is recommended to avoid excess exposure to high concentrations of estrogen during ovarian stimulation in breast cancer patients. To minimize this potential risk with super-physiological levels of serum estrogen used with controlled ovarian stimulation, it is sensible to use modified gonadotropin protocol in combination with letrozole or tamoxifen.

\section{Implications for clinical practice}

Fertility preservation is one area in which cancer service providers need to focus on developing more formal methods to inform young women with breast cancer about the impact of cancer on fertility and options for fertility preservation. Access to quality patient education, stream-lined referrals to fertility experts, and providing continuing staff education can be the initial steps in providing this level of care to young women with breast cancer. In addition, many cancer care providers have access to cancer survivorship resources, including navigators, who are well suited to facilitate education and access to resources for many community and academic cancer care practice settings. One of the challenges in educating patients on fertility preservation is the lack of adequate community resources for a timely referral. Therefore, this requires an established relationship with a fertility clinic to ensure timely consultation with young women with breast cancer interested in fertility preservation. This means not waiting weeks or months for an appointment, but scheduling within a few days. Establishing this type of network will facilitate a more efficient process and will prevent unnecessary treatment delays.

\section{Summary}

Young women diagnosed with breast cancer require a multidisciplinary approach to treatment planning, which includes access to information and resources to address fertility. Informed decision making regarding treatment options includes understanding the late and long-term effects of treatment and loss of fertility along with options for maintaining fertility should be a standard part of the discussion. The development of stream-lined referrals to qualified reproductive specialists is key to this process working due the sense of urgency by both the providers and patients to initiate the treatment process.

Understanding the needs of young women with breast cancer, making practice adjustments, and establishing relationships between oncology and reproductive specialists will make this process more efficient. Utilizing breast cancer survivorship programs and patient navigators may also help facilitate access to fertility preservation services and improve the experience of both patients and providers. 


\section{Addendum}

Fertility Preservation: Recommendations for Program Development and Patient Directed Care

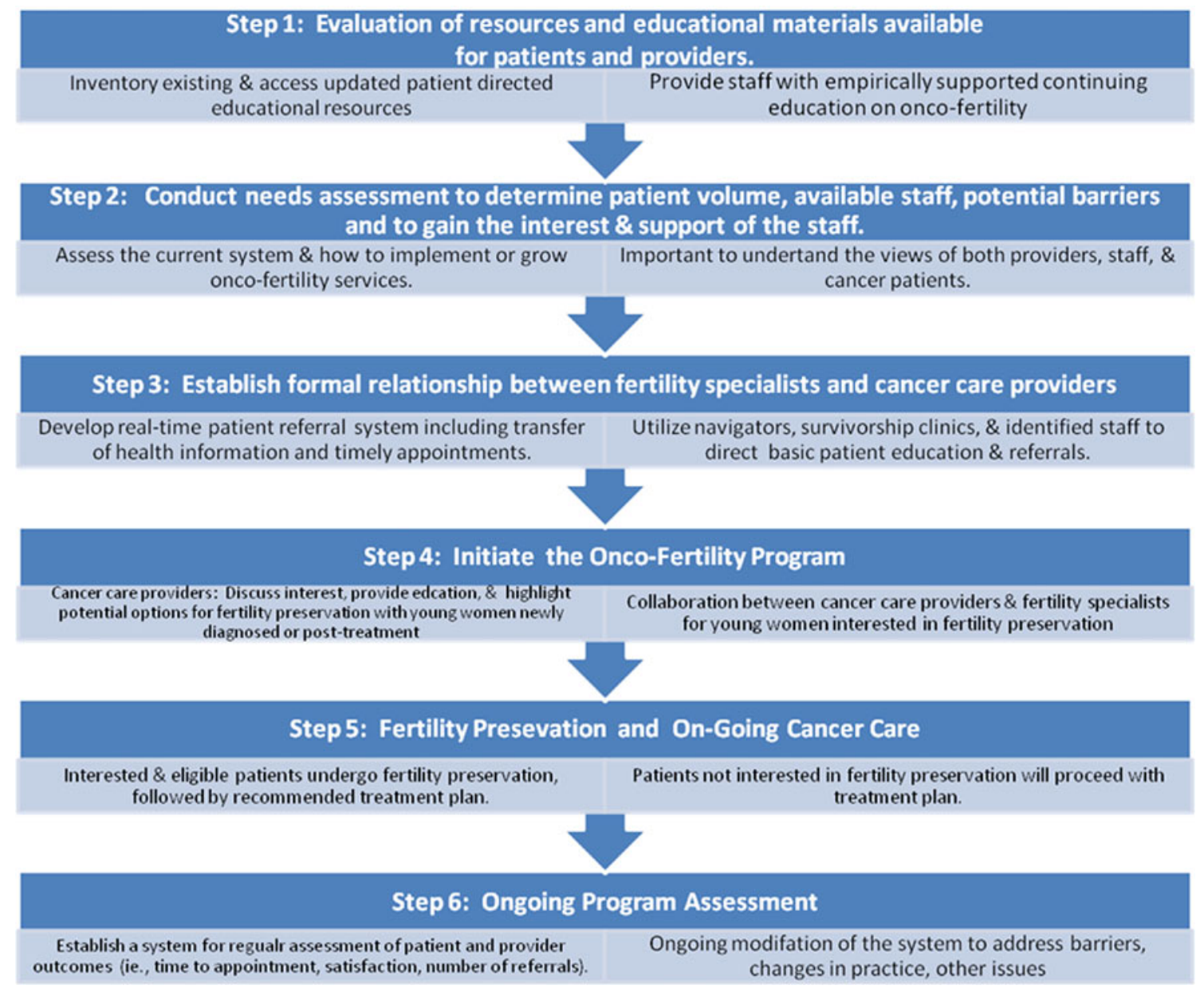

\section{References}

1. ACS Facts and Figures;2011.

2. Anders CK, Johnson R, Litton J, Phillips M, Bleyer A. Breast cancer before age 40 years. Semin Oncol. 2009; 36:237-49.

3. Hayat MJ, Howlader N, Reichman ME, Edwards BK. Cancer statistics, trends, and multiple primary cancer analyses from the Surveillance, Epidemiology, and End Results (SEER) Program. Oncologist. 2007;12:20-37.

4. Letourneau JM, Katz PP, Smith JF, Ebbel E, Cedars MI, Rosen MP. The impact of fertility counseling and fertility preservation on long-term psychosocial outcomes in young female cancer survivors. Fertil Steril. 2010;94(4):S65.

5. Lee SJ, Schover LR, Partridge AH, Patrizio P, Wallace WH, Hagerty K, Beck LN, Brennan LV, Oktay K. American Society of Clinical Oncology. The American Society of Clinical
Oncology Recommendations on Fertility Preservation in Cancer Patients. J Clin Oncol. 2006;24:2917-31.

6. Quinn G, Vadaparampil ST, Jacobsen P, Lee J, Lancaster J, Bepler $\mathrm{G}$, et al. National survey of physician practice patterns: fertility preservation and cancer patients.

7. Kim SS, Klemp JR, Fabian CJ. Breast cancer and fertility preservation. Fertil Steril. 2011;95(5):1535-43.

8. Hulvat M, Jeruss J. Fertility Preservation Options for Young Women with Breast Cancer. Curr Opin Obstet Gynecol. 2011;23(3):74-182.

9. Hickey M, Peate M, Saunders CM, Friedlander M. Breast cancer in young women and its impact on reproductive function. Hum Reprod Update. 2009;15(3):323-39.

10. von Wolff M, Montag M, Dittrich R, Denschlag D, Nawroth F, Lawrenz B. Fertility preservation in women-a practical guide to preservation techniques and therapeutic strategies in breast cancer, Hodgkin's lymphoma and borderline ovarian tumours by the fertility preservation network FertiPROTEKT. Arch Gynecol Obstet. 2011;284(2):427-35. 